\title{
GEOMETRIC-TOPOLOGIC DESCRIPTION OF A COMPLEX ROAD JUNCTION CONSIDERING THE REQUIREMENTS OF HIGHLY AUTOMATED DRIVING
}

\section{JANOS MATE LOGO ${ }^{1}$, VIVIEN POTO ${ }^{2}$, NIKOL KRAUSZ ${ }^{3}$ AND ARPAD BARSI ${ }^{4}$}

\author{
${ }^{1}$ BME Department of Photogrammetry and Geoinformatics \\ H-1111 Budapest, Muegyetem rkp. 3, Hungary \\ logo.janos.mate@emk.bme.hu, https://epito.bme.hu/logo-janosmate \\ ${ }^{2}$ BME Department of Photogrammetry and Geoinformatics \\ H-1111 Budapest, Muegyetem rkp. 3, Hungary \\ poto.vivien@emk.bme.hu, https://epito.bme.hu/poto-vivien \\ ${ }^{3}$ BME Department of Photogrammetry and Geoinformatics \\ H-1111 Budapest, Muegyetem rkp. 3, Hungary \\ krausz.nikol@emk.bme.hu, https://epito.bme.hu/krausz-nikol \\ ${ }^{4}$ BME Department of Photogrammetry and Geoinformatics \\ H-1111 Budapest, Muegyetem rkp. 3, Hungary \\ barsi.arpad@emk.bme.hu, https://epito.bme.hu/barsi-arpad
}

Key words: Highly Automated Driving, Map Standard, Complex Junction, Graph Building.

\begin{abstract}
The highly automated driving research is one of the most remarkable developments in the automotive world. Still, ordinary people can also realize the milestones facing more and more built-in vehicle assistant services brought by the new car manufacturers. The navigation system is also part of the "success story" because the maps are currently high-resolution 3D databases, and permanently better visualization supports the drivers. The creation of these map databases relies on cutting-edge field data capture techniques, like aerial surveys. The paper presents a technology based on aerial photographs' evaluation done by human operators. The captured photographs were transformed into distortion-free orthophotos; then, skilled humans evaluated the expected database elements: road and lane segments. The map creation procedure aimed to serve the content fully compatible with the standards of the industry. This evaluation procedure resulted in a road and a lane-level graph, clearly in harmony with the traffic rules. The CAD-drawings of each operator form an excellent starting point to start new artificial intelligence developments.
\end{abstract}

\section{INTRODUCTION}

One of the big technical breakthroughs of the 21 st century in transportation is expected from self-driving vehicles. The technological advances result in more and more automation even today, later the safety on roads is higher, the energy consumption is more efficient, the 
traveling time is shorter; additionally, there are fewer vehicles on roads with smaller environmental load, and more space is available for urban use.

The control of these vehicles requires more detailed maps with increased accuracy. These digital databases contain elements, which are not part of the current maps, but vehicles can use them in their environmental perception or localization procedures. The map term has been undergone a paradigm change:

1. the map content is stored in digital databases - more preferable now in a distributed way utilizing the cutting-edge technological achievements, like cloud storage and computing,

2. fully $3 \mathrm{D}$ spatial description forms the content for all stored elements, extending with unusual data sources, like point clouds, surface and volume models, spatial geometric primitives, etc.

3. accuracy has been increased significantly, which means that only the available highest quality field survey and data processing technology is applicable during the mapping procedure, as well as data quality measures are simultaneously derived and served,

4. map change detection and update workflow guarantee the up-to-dateness (currency) status for the database; even it can be achieved in the sub-second time range, enabling of handling of features with dynamics.

The listed map features have a consequence that this map differs radically from the (historically improved) navigation maps. Therefore, the "newly born" map is called a HighDefinition map, or abbreviated as HD map, opposite to the formerly created so-called Small. Definition (SD) map. Because of the obviously increased anount of data with the enlarged
complexity in their interpretation, HD map is intended for machine (computer) use, whereas
SD map suits human understanding. HD maps are commonly meant as a supporting product
in Highly Automated Driving (HAD), so HAD map term is also turning up.
It is evident that HD maps and the related technology are the results of continuous and exhaustive development. The technology for creating those content and adequate storage is an

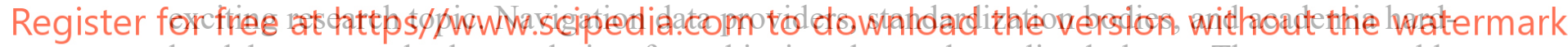
ly elaborate on the best solution for achieving the goals outlined above. The most notable standardized technologies with detailed format description documentation are (1) the Navigation Data Standard (NDS) format from the NDS Association, (2) the OpenDRIVE format from ASAM (Association for Standardization of Automation and Measuring Systems, formerly from Vires) and (3) the Geographic Data Format, abbreviated as GDF from the International Organization for Standardization (ISO). The mentioned three formats are common in their dedicated primary goal to serve with a comprehensive and precise description of the road infrastructure and neighborhood.

The current paper is based on the NDS format. Because the extent is strongly limited, we want to demonstrate the technology and its application on a complex example, so a nearby traffic junction was selected. The paper is organized as follows: Section 2 presents the requirements and supporting standards in highly automated driving, a very compact overview of the mentioned standard. Section 3 gives a simplified summary of the working methodology. Section 4 describes the pilot site, and the obtained results can be read in Section 5. Finally, a concluding section and reference literature can be found. 


\section{REQUIREMENTS AND SUPPORTING STANDARDS IN HIGHLY AUTOMATED DRIVING}

During the last two decades, the global spread of digital technologies, also called the digital revolution in the automotive industry, has changed the way maps are being created and used by the industry. Digital maps have made their way into the vehicles in the form of single-purpose personal navigation devices (PNDs), on-board, integrated navigation systems, and applications on smartphones used by vehicle drivers. In addition to the in-vehicle navigation applications, a new breed of map supported in-vehicle feature was born, the so-called map-supported Advanced Driver Assistance Systems (ADAS) such as Curve Speed Warning System and Fuel Efficiency Advisor System. However, the use of digital maps in a machine environment represents different challenges and different production approaches. This study [1] has analyzed existing standards and specifications for describing and exchanging roadrelated geospatial information from GIS to ITS databases, based on six requirements. The need for more accurate digital maps met with the emerging new mapping technologies, as discussed in the article of S.K. Govil and S. Agrawal [2].

Matthaei and Maurer [3] apply the map term in an extended meaning, i.e., with information about traffic light status, weather conditions, and movable objects, like road closures, traffic jams, and also other traffic participants, too. The sensors and actuators have their natural position in the schema. They point on the importance of the automatic map data update. They propagate the map-aided localization process but warn that the literature is still not consistent.

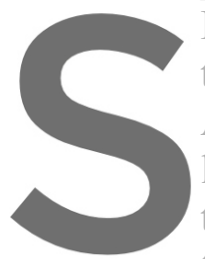
Environmental perception requires a vehicle
termination is required, later also semantic in
An absolute global position is to correct geon
lanes, or point landmarks [4]. The road netwo
tinuously by change, i.e., traffic jams, accidents,
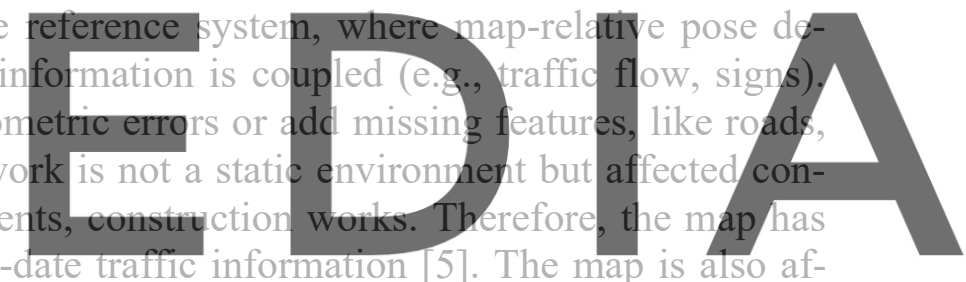

to be continuously updated to provide up-to-date traffic information [5]. The map is also affected by changes in physical and digital infrastructure [6]. All in all, the development of con-

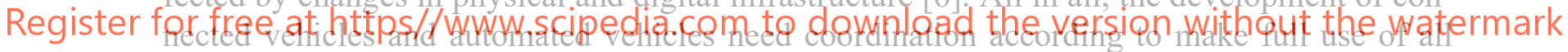

the potential benefits of fully autonomous/driverless cars [7]. The key is to have a standard to make, update, and provide HD map information.

The Navigation Data Standard (NDS) [8] effort began in 2004 and became an association registered in Germany in 2009. NDS is a standardized format for automotive-grade navigation databases, jointly developed by automobile manufacturers and suppliers whose objective is the standardization of the data format used in-car navigation systems, as well as allow a map update capability. NDS separates navigation software from navigation data. The standardization would improve interoperability and enhancing flexibility for creating various navigation products for end-users. The driver behavior is covered by traffic rules, which sensors often cannot collect themselves. For HAD, the NDS map data format offers specific building blocks that layer the data and references it to each other (Figure 1) [9]. 


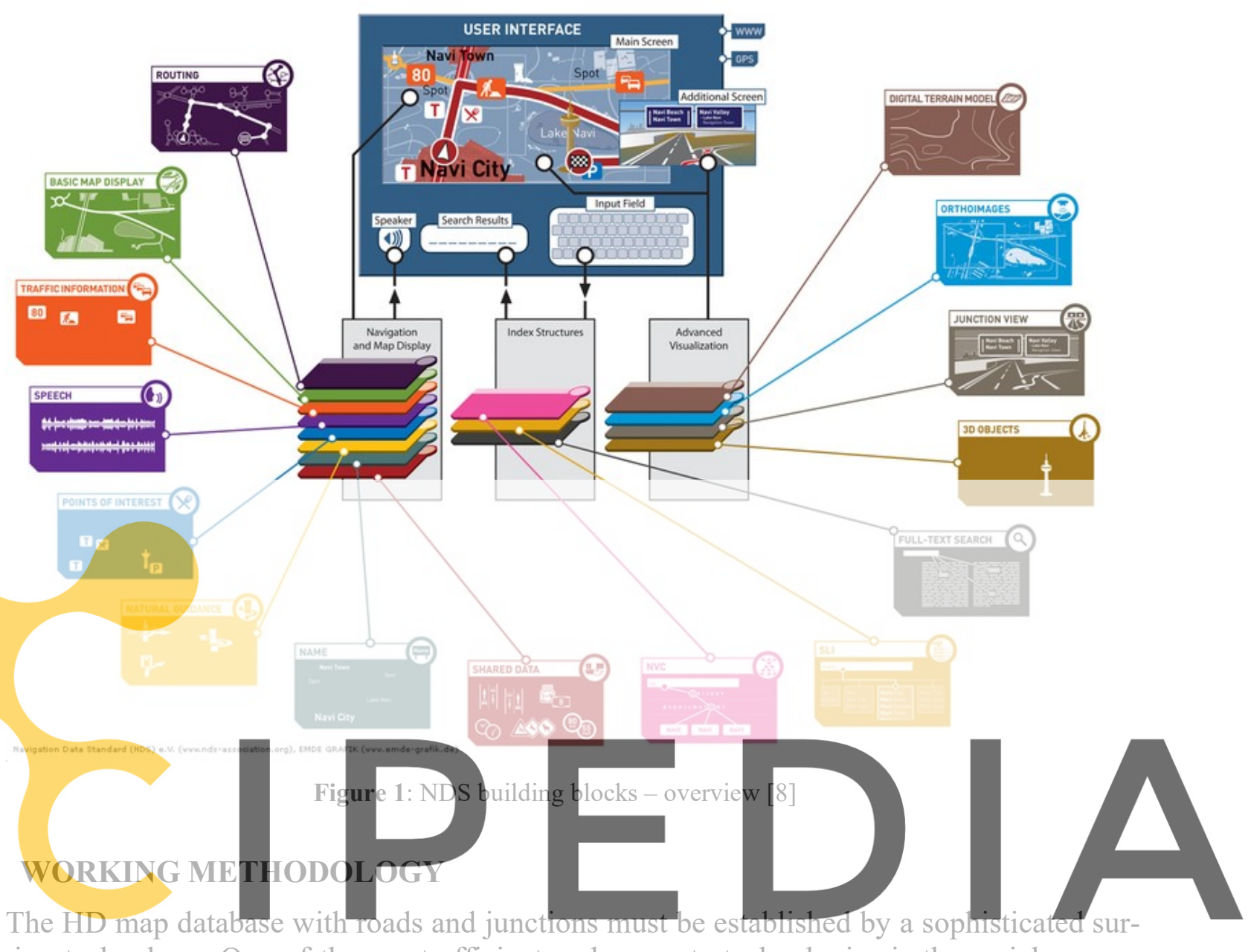

veying technology. One of the most efficient and accurate technologies is the aerial survey.

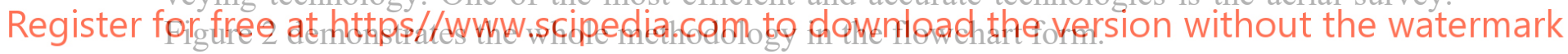

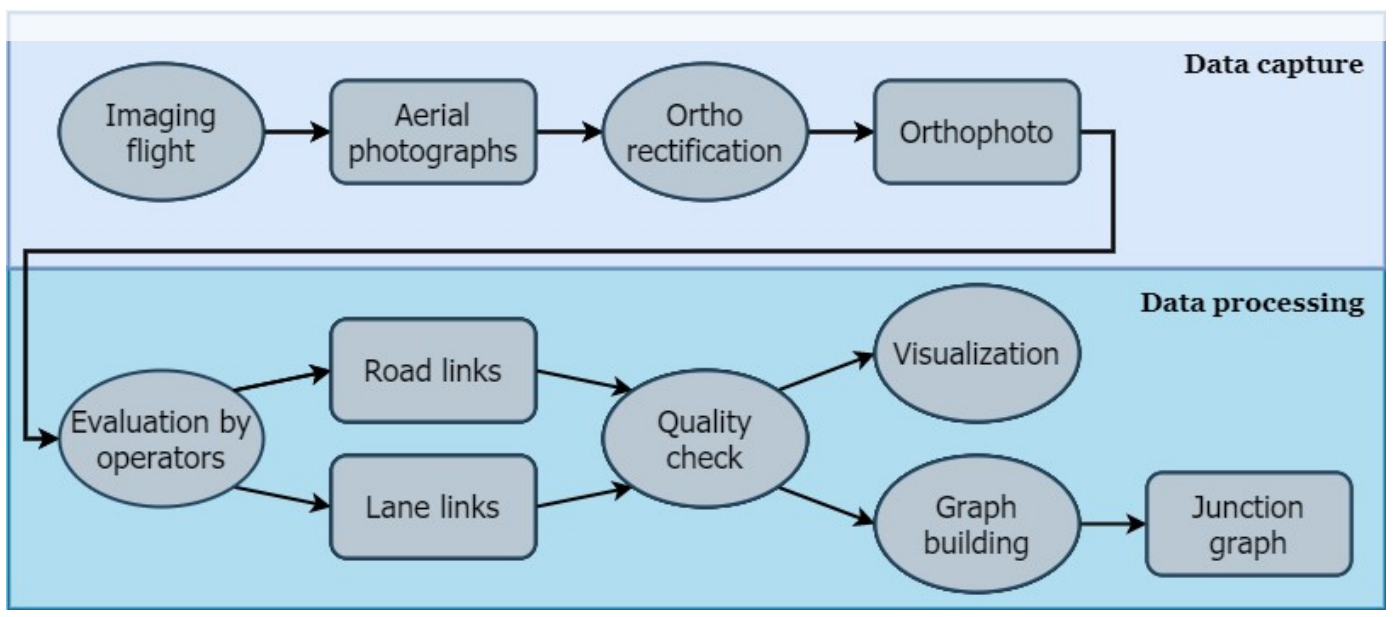

Figure 2: The implemented workflow 
The technology is built up of an airplane flight, where high-resolution color images are captured together with the necessary navigation data. The obtained image sets are then processed by photogrammetric computation called orthorectification. This method computes the precise spatial positions and orientations for all captured images, then the imagery is projected on a high-resolution digital elevation model. The created orthophoto is an image with no perspective, and height caused distortion. The geometric resolution of these orthoimages is in the $\mathrm{dm}$ range, enabling the precise evaluation concerning the HD mapping requirements.

After the data capture phase, the color orthoimagery is taken as a background, and the map content evaluation follows. In this data capture phase, the objects specified by the NDS standard are extracted. Because the aim was to create a description of a complex junction considering HD map expectations, the focus was set on the road and lane links. The NDS standard specifies how all transitions create a junction in harmony with the traffic rules. Figure 3 shows a scheme where the identifiers for roads, lanes, and connectors fulfill the requirement in an example road crossing.

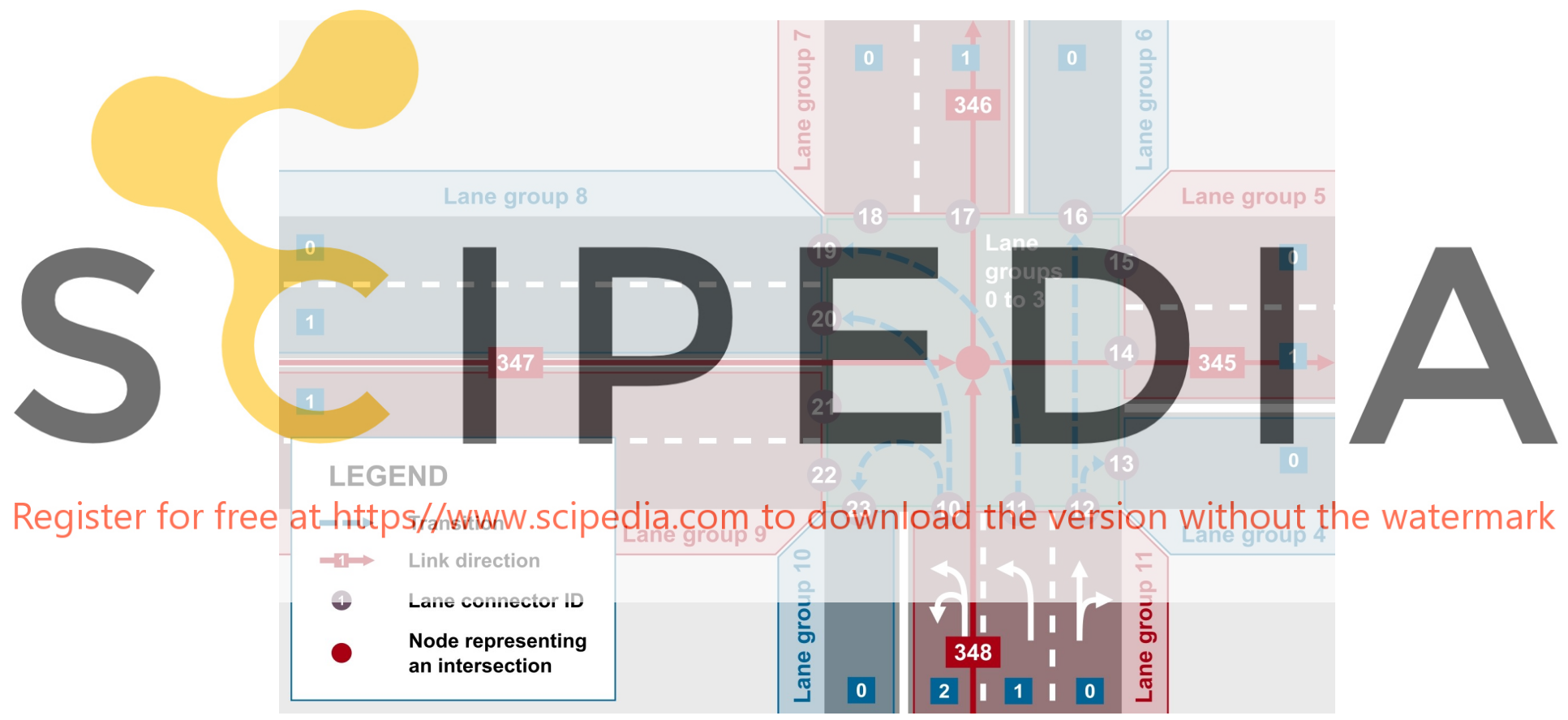

Figure 3: NDS scheme for junction elements and identifiers [10]

The requirement clearly prescribes that the road segments form a graph structure with a higher abstraction level, where the geometry expresses only the roads' connectivity. On this level, a junction is handled as a graph node. Following a top-down approach, the next level is lanes, where the roads are composed of lanes. All homogeneous regions are collected into lane groups. Such a group contains the lanes with a constant number at its starting and ending side. If new directions open or existing ones close, a new lane group is required - bringing the flexibility and ease to describe the new or disappearing lane(s). The junction center is a compound region with multiple virtual lanes forming the transitions that connect the lanes of different roads considering the traffic rules (e.g., turn around, left turn, straight direction, or right 
turn). The graph notation for the lane level is also edges and nodes, except that edges are called transitions, and nodes are the lane connectors. With these presented elements, one can build a graph description for the junction, even in complex situations. Section 5 illustrates this theory on the sample, as shown in Section 4.

The physical storage of the road and lane elements can be realized based on the above created logical scheme. We ignore this very technical explanation in our paper, but details are in NDS specification [10].

\section{SAMPLE JUNCTION}

The Szent Gellért square (Figure 4) is a busy place with several connecting roads, streets, quays, where not only public roads but also tram lines, a tram and bus station, as well as a bridge entry can be found. This area has all the complexity, which can efficiently prove the usage of the above-written standards.

The orthoimage enables the delineation of the paved and green surfaces, as well as it helps in differentiating between the lanes, sidewalks, and all elements, which are regulated in the standards. Further positive features of an orthophoto are its scale-truth (metric) representation, and of course, it is precisely georeferenced, so the derived models are ready for direct simulation inputs.
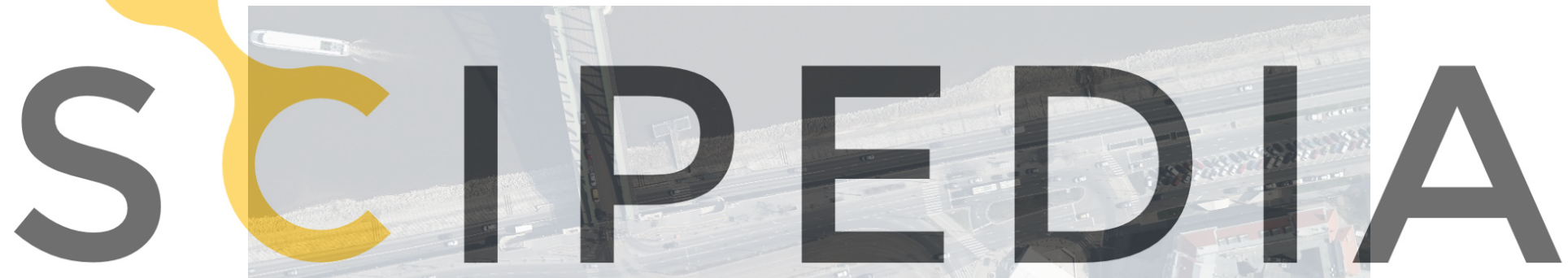

Register for free at https//www.scipedia.com to download the version without the watermark

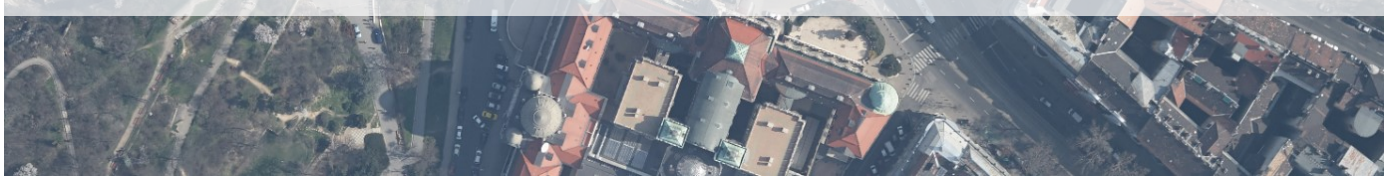

Figure 4: The pilot site of the Szent Gellért square in Budapest

\section{RESULTS}

The map content evaluation technique for HD maps is developed to create a line drawing of road and lane representation. The easiest way to make this step is with human operators with a driving license because they can recognize the situations, lanes, lane changes, etc. In all cases, when the orthophoto with the road marking has a lack of evidence, the nearby selected pilot site can be visited, and the operators can check the reality against the image information. Working at a university, these skilled operators were our master students.

One of the evaluators was responsible for extracting the road level linkage (Figure 5 part a), while others focused on the lane links. Roads express just the square's street structure, where- 
as the lanes are aimed to represent by their (quasi) center lines. After finishing the independent evaluations, all extracted content were merged into a single CAD drawing (Figure 5 part b), where we could check the quality. The primary emphasis was laid on topological accuracy (the connectivity), then completeness and geometric simplicity.

Because the map database can contain only unique real lane (transition) lines, the independent evaluations for each and every centerline had to be fused. The fusion was performed by discussing all problematic spots, so the final result was obtained with consensus (Figure 5 part c).

The next step is the finalizing of the evaluation, which is the annotation. During this phase, all road components, lanes, and lane connectors must be labeled by unique identifiers so that the junction graph elements can be accessed obviously (Figure 5 part d).
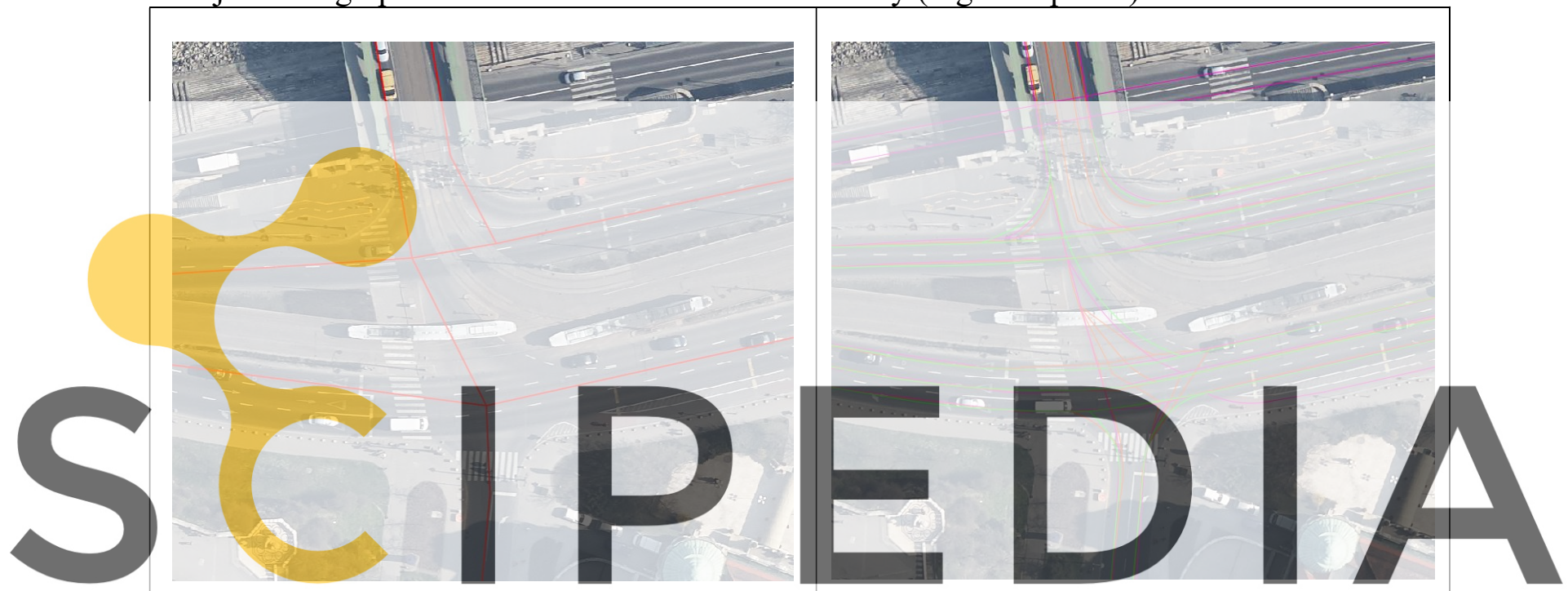

a) evaluated road links

b) lane links by all evaluators

Register for free at https//www.scipedia.com to download the version without the watermark

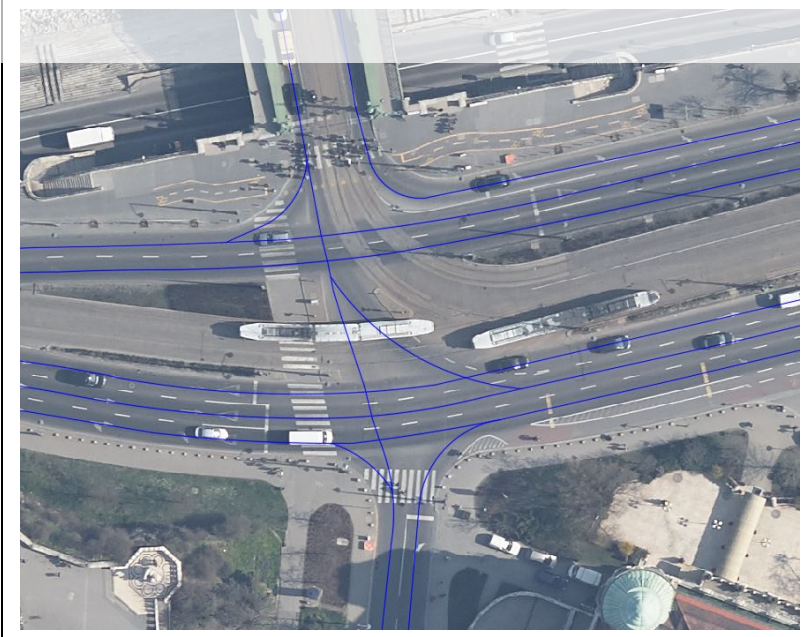

c) lane links with consensus

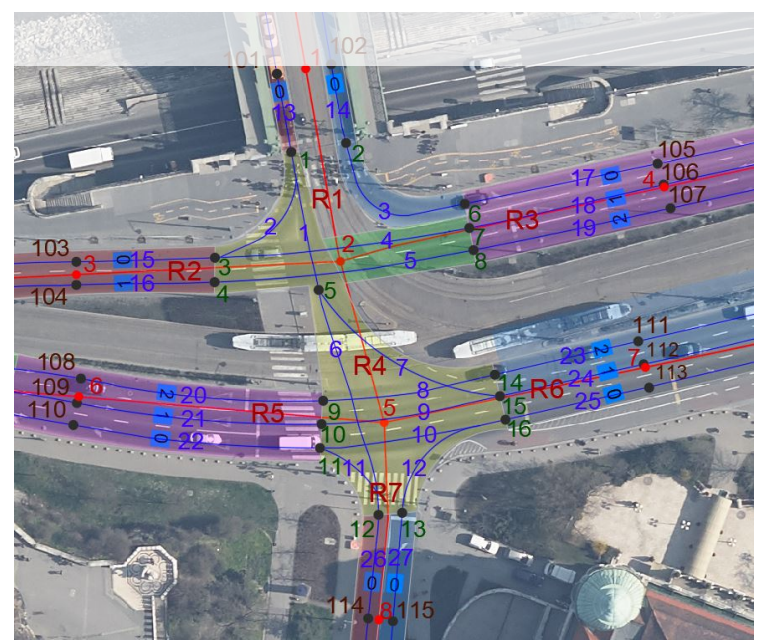

d) evaluation extended by all identifiers

Figure 5: Result of the evaluation procedure 
The quality control in our workflow was realized by involving a map product created by a highly respected map provider, the HERE Technologies. HERE is a company that was one of the pioneers in HD maps. Their HD Live Map product is overall tested and frequently used already in the development of autonomous vehicles. Figure 6 shows the same area in Live Map. Visually the two map works can be compared, and one can notice that the lane center lines and their connectivity have an excellent coincidence with our evaluation. (There is also a notable shortage in connectivity in the Live Map.)

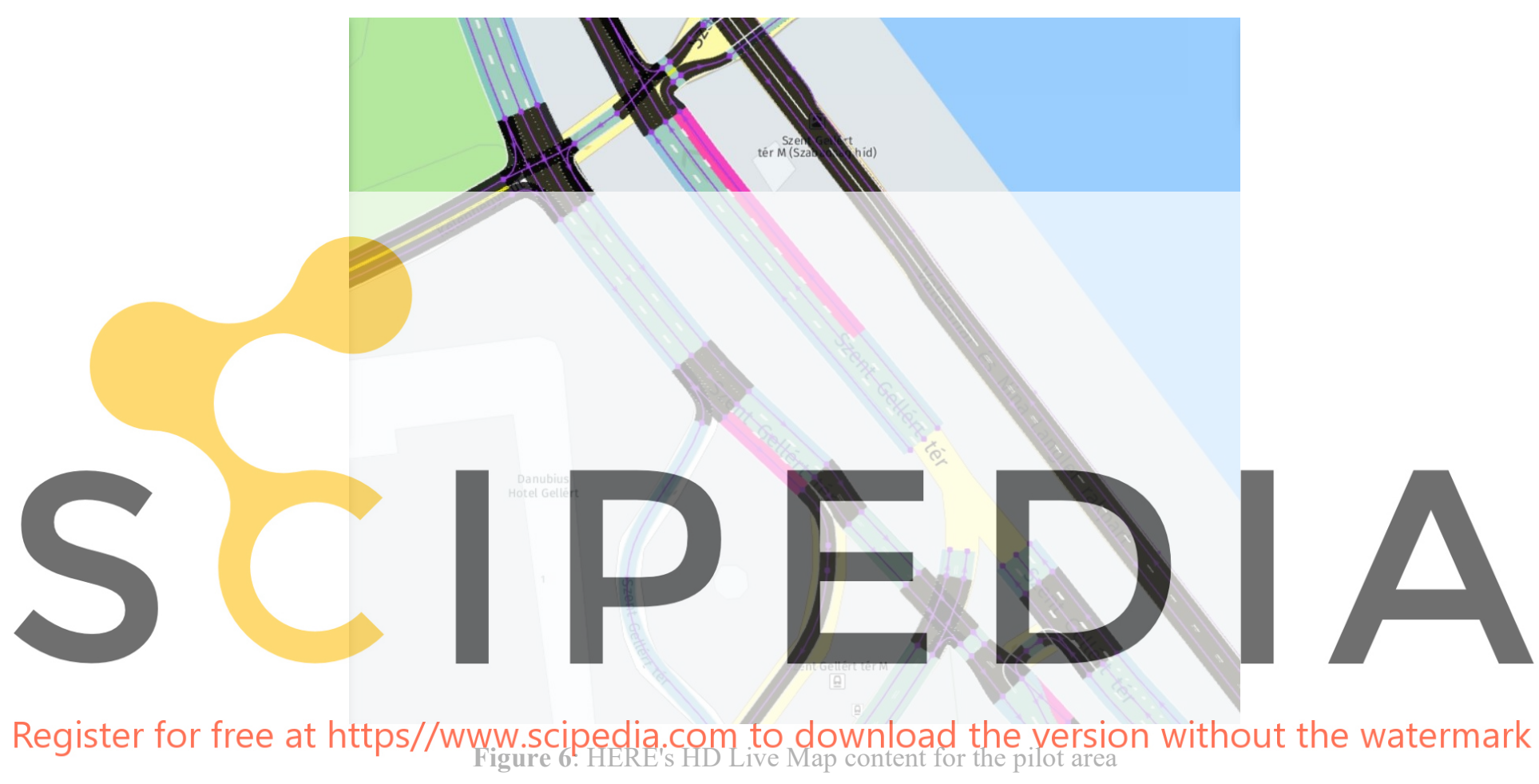

The junction graph on road level has been built from 8 points, so the adjacency matrix has a size of $8 \times 8$, but it is a sparse matrix having only 7 nonzero elements. The lane level description with a graph has 31 points forming a $31 \times 31$ adjacency matrix and having 27 lanes as nonzero elements. The sparsity with roads is $10.9 \%$ nonzeros and $2.8 \%$ for lanes.

\section{CONCLUSION}

Our understanding of maps has been significantly changed in the last decades. It is especially true for navigation, where the map plays a crucial role. The map is now interpreted as a digital database that can be stored distributed and with powerful database engines. Thanks to these tools, the update procedure has been accelerated, so rapidly changing features (e.g., road works) can also be stored consequently in this database. The content of nowadays' map is $3 \mathrm{D}$, and the relevant data quality measures are also registered. The map creation technologies can profit from the technical capabilities of field surveys, like aerial photographs or mobile mapper surveys taking part in those campaigns. 
The advantage of using images in map building tasks is the easy understanding of situations on the surface: roads, markings, buildings, vehicles indicate the road infrastructure elements, so based on their perception, an accurate evaluation methodology can be established. This technology was set up, a nearby complex region was selected for the pilot site, and the steps were realized by involving university students. The evaluation job cleared that different operators interpret the connectivity of the lanes differently, so more than one operator is needed to guarantee the high quality. After merging the evaluation versions of multiple operators, a consensus had to be found, which was kept as the result of the object extraction. After the road and lane segments' derivation, a graph structure was built, representing the main information layers for navigation purposes.

The road and lane extraction method should be automatized, although the procedures to be applied require controlled input information. If artificial intelligence, like deep learning, is aimed to be deployed, an adequate set with annotated roads and lanes must be present to the training algorithm. Similarly, reinforced learning efforts also need some information, which can be used by the system in the development of the right agent policy. The human evaluation done by our students will serve as an excellent basis to start those experiments.

\section{ACKNOWLEDGEMENT}

The research reported in this paper and carried out at the Budapest University of Technology and Economics has been supported by the National Research Development and Innovation Fund (TKP2020 Institution Excollence Subprogram, Grant No. BME-IE-VinFM) based on the charter of bolst under the auspices of the

The project has been cial Fund. EFOP-3.6.3-VEKOP-16-2017-0000 1.

REFERENCES

Register for free at https//yww.scipedia,com to download the version withput the watermark Geospatial ITS Databases Based on a Generic Model," ISPRS Int. J. Geo-Information, vol. 8, no. 3, p. 141, 2019.

[2] S. K. Govil and S. Agrawal, "Digital Mapping as an Emerging Technology in Surveying and Mapping in Urban Areas," Indian Inst. Remote Sens., pp. 105-110, 2003.

[3] R. Matthaei and M. Maurer, "Autonomous driving - A top-down-approach," AtAutomatisierungstechnik, vol. 63, no. 3, pp. 155-167, 2015.

[4] "Analysis of geospatial data requirement to support the operation of autonomous cars Project Report SDFE-Danish Ministry of Energy, Utilities and Climate,” 2017.

[5] F. Jomrich, J. Schmid, S. Knapp, A. Hos, R. Steinmetz, and B. Schuller, “Analysing communication requirements for crowd sourced backend generation of HD Maps used in automated driving," in IEEE Vehicular Networking Conference, VNC, 2019, vol. 2018-Decem.

[6] H. Farah, S. M. J. G. Erkens, T. Alkim, and B. van Arem, "Infrastructure for 
Automated and Connected Driving: State of the Art and Future Research Directions," 2018, pp. 187-197.

[7] EUROPEAN COMMISSION, "Roadmap on Highly Automated vehicles," 2016.

[8] “NDS Association webpage," 2018. [Online]. Available: https://www.ndsassociation.org/. [Accessed: 29-Oct-2018].

[9] B. Philip Hubertus with contributions by Martin Schleicher, F. Klebert, G. Horn, and M. Junker, "NDS: The Benefits of a Common Map Data Standard for Autonomous Driving," 2019.

[10] "NDS Open Lane Model webpage," 2019. [Online]. Available: http://www.openlanemodel.org/. [Accessed: 31-Jan-2019].
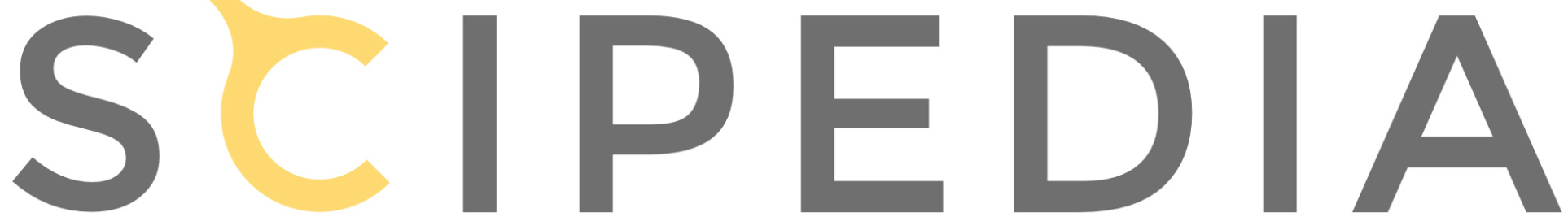

Register for free at https//www.scipedia.com to download the version without the watermark 